\title{
Early venous thromboembolism chemoprophylaxis in combat-related penetrating brain injury
}

\author{
R. Michael Meyer, BS, ${ }^{1}$ M. Benjamin Larkin, PharmD,, Nicholas S. Szuflita, MPH, ${ }^{1}$ \\ Chris J. Neal, MD, ${ }^{2}$ Jeffrey M. Tomlin, MD, ${ }^{2,3}$ Rocco A. Armonda, MD, ${ }^{2,4}$ Jeffrey A. Bailey, MD, ${ }^{5}$ and \\ Randy S. Bell, MD2
}

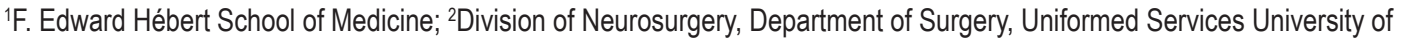
the Health Sciences, Walter Reed National Military Medical Center, Bethesda, Maryland; ${ }^{3}$ Department of Neurosurgery, Naval Medical Center San Diego, California; ${ }^{4}$ Department of Neurosurgery, Georgetown University, Washington, DC; and ${ }^{5}$ Department of Surgery, Uniformed Services University of the Health Sciences, Walter Reed National Military Medical Center, Bethesda, Maryland
\end{abstract}

\begin{abstract}
OBJECTIVE Traumatic brain injury (TBI) is independently associated with deep vein thrombosis (DVT) and pulmonary embolism (PE). Given the numerous studies of civilian closed-head injury, the Brain Trauma Foundation recommends venous thromboembolism chemoprophylaxis (VTC) after severe TBI. No studies have specifically examined this practice in penetrating brain injury (PBI). Therefore, the authors examined the safety and effectiveness of early VTC after PBI with respect to worsening intracranial hemorrhage and DVT or PE.
\end{abstract}

METHODS The Kandahar Airfield neurosurgery service managed 908 consults between January 2010 and March 2013. Eighty of these were US active duty members with PBI, 13 of whom were excluded from analysis because they presented with frankly nonsurvivable CNS injury or they died during initial resuscitation. This is a retrospective analysis of the remaining 67 patients.

RESULTS Thirty-two patients received early VTC and 35 did not. Mean time to the first dose was 24 hours. Fifty-two patients had blast-related PBI and 15 had gunshot wounds (GSWs) to the head. The incidence of worsened intracranial hemorrhage was $16 \%$ after early VTC and $17 \%$ when it was not given, with the relative risk approaching $1(R R=0.91)$. The incidence of DVT or PE was $12 \%$ after early VTC and $17 \%$ when it was not given $(R R=0.73)$, though this difference was not statistically significant.

CONCLUSIONS Early VTC was safe with regard to the progression of intracranial hemorrhage in this cohort of combatrelated PBI patients. Data in this study suggest that this intervention may have been effective for the prevention of DVT or PE but not statistically significantly so. More research is needed to clarify the safety and efficacy of this practice. https://thejns.org/doi/abs/10.3171/2016.4.JNS16101

KEY WORDS penetrating brain injury; venous thromboembolism chemoprophylaxis; military neurosurgery; trauma; traumatic brain injury

$\mathrm{T}$ HE association between traumatic brain injury (TBI) and hypercoagulability is well established, but venous thromboembolism (VTE) chemoprophylaxis (VTC) strategies remain controversial. Traumatic brain injury itself may confer as much as a 4-fold increased risk of deep vein thrombosis (DVT) in trauma patients. ${ }^{13}$ In a study of 88 TBI patients with admission Glasgow Coma Scale (aGCS) scores consistent with the full spectrum of TBI (mild, moderate, and severe), there was a $25 \%$ inci- dence of DVT, ${ }^{6}$ and up to one-third of moderate to severe TBI patients have been found to suffer DVT. ${ }^{8}$ Observational studies show that VTC may decrease the incidence of DVT and VTE among patients with TBI without increasing the progression of intracranial hemorrhages (ICHs) in those with stable head CT studies and/or neurological examinations. ${ }^{9,14}$ The Delayed Versus Early Enoxaparin Prophylaxis I (DEEP-I) randomized control trial found that ICH progression rates among TBI patients with stable

ABBREVIATIONS aGCS = admission Glasgow Coma Scale; aRR = adjusted relative risk; AIS = Abbreviated Injury Scale; DVT = deep vein thrombosis; GSW = gunshot wound (to the head); ICH = intracranial hemorrhage; ISS = Injury Severity Score; KAF = Kandahar Airfield; PBI = penetrating brain injury; PE = pulmonary embolism; RR = relative risk; TBI = traumatic brain injury; TXA = tranexamic acid; UFH = unfractionated heparin; USAISR = US Army Institute of Surgical Research; VTC = VTE chemoprophylaxis; VTE = venous thromboembolism.

SUBMITTED January 13, 2016. ACCEPTED April 7, 2016.

INCLUDE WHEN CITING Published online June 17, 2016; DOI: 10.3171/2016.4.JNS16101. 
hemorrhages who had received early VTC were similar to those in patients who had been treated with placebo. ${ }^{12}$

The 2007 Brain Trauma Foundation severe TBI guidelines recommended VTC with low molecular weight heparin or unfractionated heparin (UFH). ${ }^{5}$ However, there is currently insufficient evidence to support the selection of a particular agent, dose, or timing for administration. ${ }^{5}$ A 2014 systematic review of over 20 studies also found that VTC is safe in TBI patients with stable hemorrhages, though the authors were unable to definitively comment on agent selection, dosage, first dose timing, or efficacy in preventing VTE. ${ }^{15}$

We have previously described the spectra and mechanisms of brain injury, and their related complications, suffered during the conflicts in Iraq and Afghanistan. ${ }^{1-4,7,16,17}$ These injuries and the circumstances surrounding the theater and medical evacuation care posed additional challenges for physicians caring for the wounded. Specifically, from 2003 to 2008, an approximately 7\% incidence of DVT or pulmonary embolism (PE) was observed within the combat-induced severe TBI population. ${ }^{3}$ The extended air evacuation flight from the theater to definitive medical care within the continental US was hypothesized to be at least partially responsible..$^{10}$

The extant literature concerning the use of chemical DVT prophylaxis in TBI is based largely or entirely on the civilian closed-head injury population. We are unaware of any study that has investigated the safety or efficacy of early VTC in patients with penetrating brain injury (PBI), whether civilian or military. While available evidence shows that the risks of VTE and its associated morbidity and mortality are great and apparently increase with worsening severity (PBI, generally, being very severe), the manner in which these factors should affect the approach to a patient with PBI is not known, and very little is definite regarding best management practices. With this in mind, we present our experience with the use of early chemical DVT prophylaxis in a population of patients suffering from PBI.

\section{Methods}

\section{Study Design and Data Collection}

We conducted a retrospective cohort study of all US military personnel for whom neurosurgical consults were made at the Multinational Medical Unit at Kandahar Airfield (KAF) in Afghanistan between January 2010 and March 2013. The US Army Institute of Surgical Research (USAISR) trauma registry was queried for a list of all patients seen by the neurosurgery service at KAF during the study period. This database included patient identifying information, mechanism of injury (penetrating or blunt), national affiliation (to include military service component if the patient was a US active duty member), Injury Severity Score (ISS), Abbreviated Injury Scale (AIS) score for the head, aGCS score, and exposure to other agents that could influence coagulability (tranexamic acid [TXA] or factor VII).

The patient list from the USAISR registry was sorted to include only US active duty service members suffering from penetrating injuries. The results were then cross- referenced with the Theater Medical Data Store (TMDS) to exclude patients who had sustained head, neck, or facial trauma that did not violate the calvaria, leaving only those with PBIs. The TMDS contains free text initial assessments, operative notes, daily progress notes, radiology reports, and pharmacy records. These items were examined for each patient, and data including the exposures of interest as well as the presenting ICH type and location were extracted and recorded by one of the authors (R.M.M.).

Another author (M.B.L.), who was blinded to the collection of exposure and initial ICH data, examined the electronic medical records from inpatient and outpatient encounters for each patient after evacuation from Afghanistan to record outcomes of interest. This study was conducted in accordance with a protocol approved by the Walter Reed National Military Medical Center Institutional Review Board.

\section{Exposures and Outcomes}

The primary exposures of interest included the use of VTC, timing of VTC administration after injury, agent(s) used, and doses administered. "Early" VTC administration was defined as the delivery of an anticoagulant agent within 48 hours of injury. The primary outcomes of interest included worsening ICH and rates of DVT and PE. Additional outcomes included emergent nonelective reoperation and early (30 days postinjury) mortality.

\section{Statistical Analysis}

Data were combined into a single data set and assessed for completeness of both exposure and outcome data. Complete outcome and exposure data were available for all patients in the cohort. One author (R.M.M.) created a de-identified data set and sent it to a third blinded investigator (N.S.S.) for analysis.

Demographic, mechanism of injury, and intracranial pathology data were analyzed according to whether or not patients received early VTC. Statistical associations were determined using the Student t-test for continuous variables, including age, ISS, AIS score, and aGCS score, and Fisher's exact test for categorical variables, including military service, mechanism of injury (that is, blast vs gunshot wound [GSW] to the head), ICH type, and presence of other intracranial pathology (for example, herniation, retained fragment, or vascular injury). Similarly, the proportion of individuals who received early VTC, the agents used for this anticoagulation, and the reasons early VTC was withheld were analyzed according to the mechanism of injury by using Fisher's exact test. The Student t-test was used to analyze the time between injury and first dose of VTC, according to the mechanism of injury.

The incidence of worsening ICH, DVT or PE, emergent reoperation, and mortality were calculated, and simple logistic regression was used to estimate the relative risk (RR) of each outcome according to whether a given patient received early VTC or not. Multiple logistic regressions were performed to estimate adjusted RRs (aRR) by adding mechanism of injury, aGCS score, ISS, and AIS score to the regression models.

A post hoc subgroup analysis was performed to inves- 


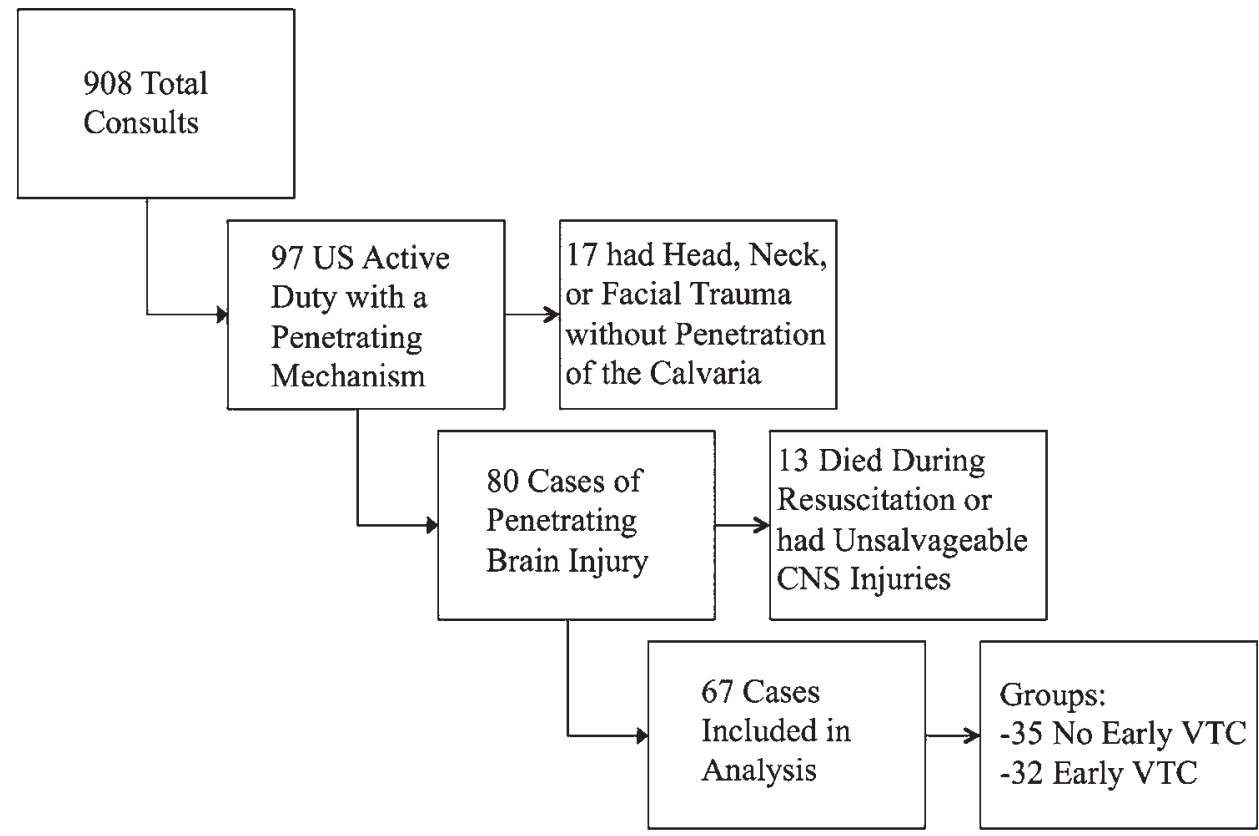

FIG. 1. Cohort inclusion and exclusion.

tigate the effect of early VTC in the blast subgroup given the preponderance of this mechanism of injury. Associations between demographic and injury characteristics were analyzed using the Student t-test and Fisher's exact test as well as logistic regression models as described above. The threshold for statistical significance was set at a $5 \%$ probability of committing a Type I error $(\alpha)$, or $\mathrm{p}<0.05$. Relative risks were considered to reflect a significant difference between groups if their associated $95 \%$ confidence interval did not include 1 . All analyses were performed using SAS version 9.3 (SAS Institute Inc.).

\section{Results}

\section{Study Population}

Figure 1 displays the results of applying the inclusion and exclusion criteria to the sample population. A total of 908 consults were made to the KAF neurosurgery service between January 2010 and March 2013. One hundred fifty-six (17\%) of these consults were for active duty US military service members. The remaining 752 consults were for a variety of patient types: $6 \%$ were allied NATO military members, $24 \%$ were allied non-NATO military members (overwhelmingly Afghan National Army and Afghan National Police), 24\% were Afghan civilians who were seen for humanitarian reasons or considered foreign civilian dependents of the US military, and 29\% did not have a specified category (including Afghan civilians who were not identifiable, enemy combatants, and others). These patients were excluded because of an inability to ascertain data about subsequent clinical courses or outcomes.

Of the US military personnel included in the study population, 97 (62\%) had sustained penetrating head injuries. However, on closer examination of the available data, 17 patients (18\%) had sustained penetrating injuries to the head, neck, or face that did not violate the inner table of the calvaria and thus were subsequently excluded. The remaining 80 patients with $\mathrm{PBI}$ represented the population eligible for inclusion in the study cohort. Of these, $13(16 \%)$ died during initial resuscitation or were deemed to be unsalvageable by the neurosurgery consultant. Thus, the final cohort consisted of 67 US active duty service members who had suffered PBIs managed at KAF during the study period.

\section{Demographics and Injury Profile}

Table 1 displays the demographic and injury characteristics of the study population. Of those who survived long enough to be considered for early VTC (67 patients), $35(52 \%)$ did not receive early anticoagulation, whereas $32(48 \%)$ did. All patients were male. The mean age of the study population overall was 25 years (range 19-38 years), and the mean age between the groups was similar (no early VTC: 25.2 years, 19-38 years; early VTC: 24.9 years, 19-37 years). Though the difference between the 2 groups was not statistically significant, the group that did not receive early VTC had worse ISSs (27.7 vs 24.8), AIS scores (4.1 vs 3.8), and aGCS scores (8.3 vs 10.3) than the group that received early VTC.

There were also no statistically significant differences in the distribution of injury types between the 2 treatment groups: a greater proportion of the group without early VTC sustained intraparenchymal hemorrhages $(83 \%$ vs $66 \%, \mathrm{p}=0.16)$, subarachnoid hemorrhages $(60 \%$ vs $34 \%$, $\mathrm{p}=0.051)$, subdural hematomas $(46 \%$ vs $22 \%, \mathrm{p}=0.07)$, major vascular injuries ( $14 \%$ vs $6 \%, \mathrm{p}=0.43$ ), and epidural hematomas $(11 \%$ vs $0, p=0.12)$. Note that for this study major vascular injury is defined as injury of the internal carotid artery; proximal anterior, middle, or posterior cerebral arteries; vertebral artery; basilar artery; superior 
TABLE 1. Demographics and clinical features of males with PBI, according to early VTC

\begin{tabular}{|c|c|c|c|c|}
\hline Variable & Total & No Early VTC & Early VTC & $p$ Value \\
\hline Total no. of patients & 67 & $35(52 \%)$ & $32(48 \%)$ & \\
\hline Mean age in yrs (range) & $25(19-38)$ & $25.2(19-38)$ & $24.9(19-37)$ & $0.7973^{*}$ \\
\hline Branch of service & & & & $0.4241 \dagger$ \\
\hline Army & $41(61 \%)$ & $23(66 \%)$ & $18(56 \%)$ & \\
\hline Navy & $2(3 \%)$ & $2(6 \%)$ & 0 & \\
\hline Marines & $18(27 \%)$ & $8(23 \%)$ & $10(31 \%)$ & \\
\hline Air Force & $6(9 \%)$ & $2(6 \%)$ & $4(13 \%)$ & \\
\hline ISS (range) & $26.3(9-59)$ & $27.7(9-59)$ & $24.8(11-50)$ & $0.2754^{*}$ \\
\hline AIS score, region 1 (range) & $4(3-5)$ & $4.1(3-5)$ & $3.8(3-5)$ & $0.2934^{*}$ \\
\hline aGCS score (range) & $9.4(3-15)$ & $8.3(3-15)$ & $10.3(3-15)$ & $0.1971^{*}$ \\
\hline Mechanism of injury & & & & $0.2496 \dagger$ \\
\hline Blast & $52(78 \%)$ & $25(71 \%)$ & $27(84 \%)$ & \\
\hline GSW & $15(22 \%)$ & $10(29 \%)$ & $5(16 \%)$ & \\
\hline \multicolumn{5}{|l|}{ Injury type } \\
\hline Intraparenchymal hematoma & $50(75 \%)$ & $29(83 \%)$ & $21(66 \%)$ & $0.1598 \dagger$ \\
\hline Subarachnoid hemorrhage & $32(48 \%)$ & $21(60 \%)$ & $11(34 \%)$ & $0.0506 \dagger$ \\
\hline Retained fragments, bone, or missile & $32(48 \%)$ & $18(51 \%)$ & $14(44 \%)$ & $0.6267 \dagger$ \\
\hline Subdural hematoma & $23(34 \%)$ & $16(46 \%)$ & $7(22 \%)$ & $0.0703 \dagger$ \\
\hline Major vascular injury & $7(10 \%)$ & $5(14 \%)$ & $2(6 \%)$ & $0.4307 \dagger$ \\
\hline Intraventricular hemorrhage & $5(7 \%)$ & $3(9 \%)$ & $2(6 \%)$ & $1 \dagger$ \\
\hline Epidural hematoma & $4(6 \%)$ & $4(11 \%)$ & 0 & $0.1152 \dagger$ \\
\hline Herniation (uncal or tonsillar) & $5(7 \%)$ & $2(6 \%)$ & $3(9 \%)$ & $0.6639 \dagger$ \\
\hline
\end{tabular}

sagittal, cavernous, straight, or transverse-sigmoid sinuses; or torcula. Blast was the most common mechanism of injury overall (78\%), but the mechanism of injury varied between the groups as well, with a greater proportion of individuals without early VTC having sustained GSWs (29\% vs $16 \%$ ), though it was not a statistically significant association $(\mathrm{p}=0.25)$.

We considered mechanism of injury as a potential source of confounding by analyzing demographics, mortality, and injury characteristics according to whether the 80 patients with PBI suffered a blast injury or a GSW to the head (Table 2). Mortality before consideration of early VTC was similar between groups (6 in the blast group, 7 in the GSW group, $\mathrm{p}=0.56$ ), but injuries due to GSW were associated with a statistically significant worse AIS score (4.5 vs 3.9, $\mathrm{p}=0.0053$ ) and aGCS score (5.4 vs 9.8; $\mathrm{p}=$ $0.0036)$ than PBIs from blasts. A trend toward a worse ISS was observed for the blast-injured group, though it was not statistically significant (27.7 vs $23.7, \mathrm{p}=0.12$ ). More GSWinjured patients were noted to have major vascular injury $(14 \%$ vs $7 \%, p=0.39)$ or transtentorial or tonsillar herniation $(32 \%$ vs $14 \%, \mathrm{p}=0.11)$, but these associations were not statistically significant.

\section{Use of Early VTC}

We considered the use of early VTC and the reasons early VTC was withheld, according to the mechanism of injury (Table 3). Early VTC use was more common among blast-injured patients ( $84 \%$ vs $16 \%, p=0.25)$, and the most commonly used agent was enoxaparin (30 mg subcutaneously, twice daily; 91\%). The other 3 patients who received early VTC were given UFH (5000 units subcutaneously, 3 times daily). None of the patients who sustained a GSW received UFH. The mean time to the first dose of VTC was $24.5 \mathrm{hrs}$ for enoxaparin (range 12-48 hrs) and 17.7 hrs (range 12-24 hrs) for UFH. There was no significant difference in the time to first dose between the blast and GSW groups $(p=0.18)$.

Of those individuals who did not receive early VTC, $71 \%$ had sustained PBI from a blast. Possible reasons for withholding VTC included worsening ICH at baseline (that is, enlarging hemorrhages on a head CT before the decision to administer or hold VTC was made), hemorrhage from an extracranial site (for example, a splenic laceration managed nonoperatively), a major intracranial vascular injury as defined above, a retained fragment abutting the internal carotid artery, and coagulopathy not corrected by resuscitation. The most common reason for withholding VTC was "not specified" (46\%), and the rates were similar between the blast- and GSW-injured group. Of note, this not-specified group includes a single individual whose neurosurgery consult note indicated that VTC should be held because of a "large intracranial hemorrhage," though this lesion was not enlarging on head CT. Ten individuals 
TABLE 2. Demographics and clinical features of males with PBI, according to mechanism of injury

\begin{tabular}{lcccc}
\hline \multicolumn{1}{c}{ Variable } & Total & Blast & GSW & p Value \\
\hline Total no. of patients & 80 & $58(73 \%)$ & $22(28 \%)$ & $0.5207^{*}$ \\
\hline Mean age in yrs (range) & $24.9(19-40)$ & $25.1(19-40)$ & $24.4(19-38)$ & $0.1232^{*}$ \\
\hline ISS (range) & $26.6(9-59)$ & $27.7(10-59)$ & $23.7(9-38)$ & $0.0053^{*}$ \\
\hline AIS score, region 1 (range) & $4.1(3-5)$ & $3.9(3-5)$ & $4.5(3-5)$ & $0.0036^{*}$ \\
\hline aGCS score (range) & $8.5(3-15)$ & $9.8(3-15)$ & $5.4(3-15)$ & $0.5643 \dagger$ \\
\hline Patient died before VTC & $13(16 \%)$ & $6(10 \%)$ & $7(32 \%)$ & $0.5643 \dagger$ \\
\hline Injury type & & & $18(82 \%)$ & $0.8051 \dagger$ \\
\hline Intraparenchymal hematoma & $60(75 \%)$ & $42(72 \%)$ & $9(41 \%)$ & $0.3232 \dagger$ \\
\hline Subarachnoid hemorrhage & $35(44 \%)$ & $26(45 \%)$ & $12(55 \%)$ & $0.7923 \dagger$ \\
\hline Retained fragments, bone, or missile & $36(45 \%)$ & $24(41 \%)$ & $6(27 \%)$ & $0.3865 \dagger$ \\
\hline Subdural hematoma & $24(30 \%)$ & $18(31 \%)$ & $3(14 \%)$ & $1 \dagger$ \\
\hline Major vascular injury & $7(9 \%)$ & $4(7 \%)$ & $1(5 \%)$ & $0.6117 \dagger$ \\
\hline Intraventricular hemorrhage & $5(6 \%)$ & $4(7 \%)$ & $2(9 \%)$ & $0.1054 \dagger$ \\
\hline Epidural hematoma & $5(6 \%)$ & $3(5 \%)$ & $7(32 \%)$ & \\
\hline Herniation (uncal or tonsillar) & $15(19 \%)$ & $8(14 \%)$ & & \\
\hline * Student t-test. & & &
\end{tabular}

were noted to have worsening ICHs at the time VTC administration was considered; 6 had PBI from blast, and 4 from GSW. These 10 patients did not receive early VTC.

Supplemental Table 1 presents the results of a post hoc subgroup analysis of demographic features and injury characteristics of individuals who did not receive early VTC, according to the reason early VTC was withheld. No statistically significant associations were found.

\section{Primary and Secondary Outcomes}

Table 4 displays the primary outcomes of interest, according to whether patients received early anticoagulation (Supplemental Table 3 for the blast subgroup). There were 11 cases $(16 \%)$ of incident-worsened ICH. The 10 individuals who did not receive early VTC because of worsening ICH in Afghanistan were considered to be at risk for new $\mathrm{ICH}$, so they are included in the denominator of this figure. Four of these 10 patients had incremental enlargement of the same lesions that were noted to have worsened before the decision to administer or hold early VTC was made, but the interval change was slight and expected; thus, these cases are not considered incident and were not included in the numerator of this figure in the group with incident-worsened ICH group. The RR of incident-worsened ICH for those who received early VTC was 0.91 (95\% CI $0.28-2.99)$. The 4 cases of worse ICH that were not considered incident are accounted for in the figures for "all worse ICH" in Table 4, and when considering these cases the incidence increases to 15 (22\%). This decreases the RR of worsened ICH for those who received early VTC to 0.55 (95\% CI $0.19-1.60)$.

There were 10 cases $(15 \%)$ of DVT or PE, with $6(17 \%)$ occurring in patients who did not receive early VTC and $4(12 \%)$ in patients who did receive it. Two of these 10 cases were PEs, and both occurred in patients who did not receive early VTC; the other 8 were isolated DVTs. This difference was not statistically significant between the groups (RR $0.73,95 \%$ CI $0.21-2.58$ ). The 30 -day mortality rate $(6 \%)$ and rate of emergent reoperation $(6 \%)$ were also similar between groups. After adjusting for mechanism of injury, aGCS score, ISS, and AIS score in the regression model, there was still no increased risk of incident or overall worsened ICH, emergent reoperation, or mortality with the use of early VTC; and still, no significant difference was observed in the rate of DVT or PE between groups.

\section{Factor VII and TXA Exposure}

Of the 67 patients in the cohort, $4(6 \%)$ received factor VII during resuscitation at KAF and 7 (10\%) received TXA. No patients received both. None of the patients receiving factor VII experienced an outcome of interest (worsened ICH or a DVT/PE). Five of the patients who received TXA experienced an outcome of interest. Two of these patients suffered a hypercoagulable complication (1 DVT and $1 \mathrm{PE}$ ); neither of them had received early VTC. Two patients had worsening ICH; neither of them had received early VTC. The fifth patient suffered both a DVT and a worsened ICH; this patient had received early VTC (enoxaparin).

\section{Subgroup Analysis}

Given the preponderance of blast mechanism of injury in this cohort, we performed a post hoc subgroup analysis of the 52 patients with blast-induced PBI. Supplemental Table 2 shows demographic and injury profile data for these patients according to whether they received early VTC. In general, the data presented here are similar to those summarized in Table 1 for the cohort overall. Analy- 
TABLE 3. Use of early VTC, according to mechanism of injury

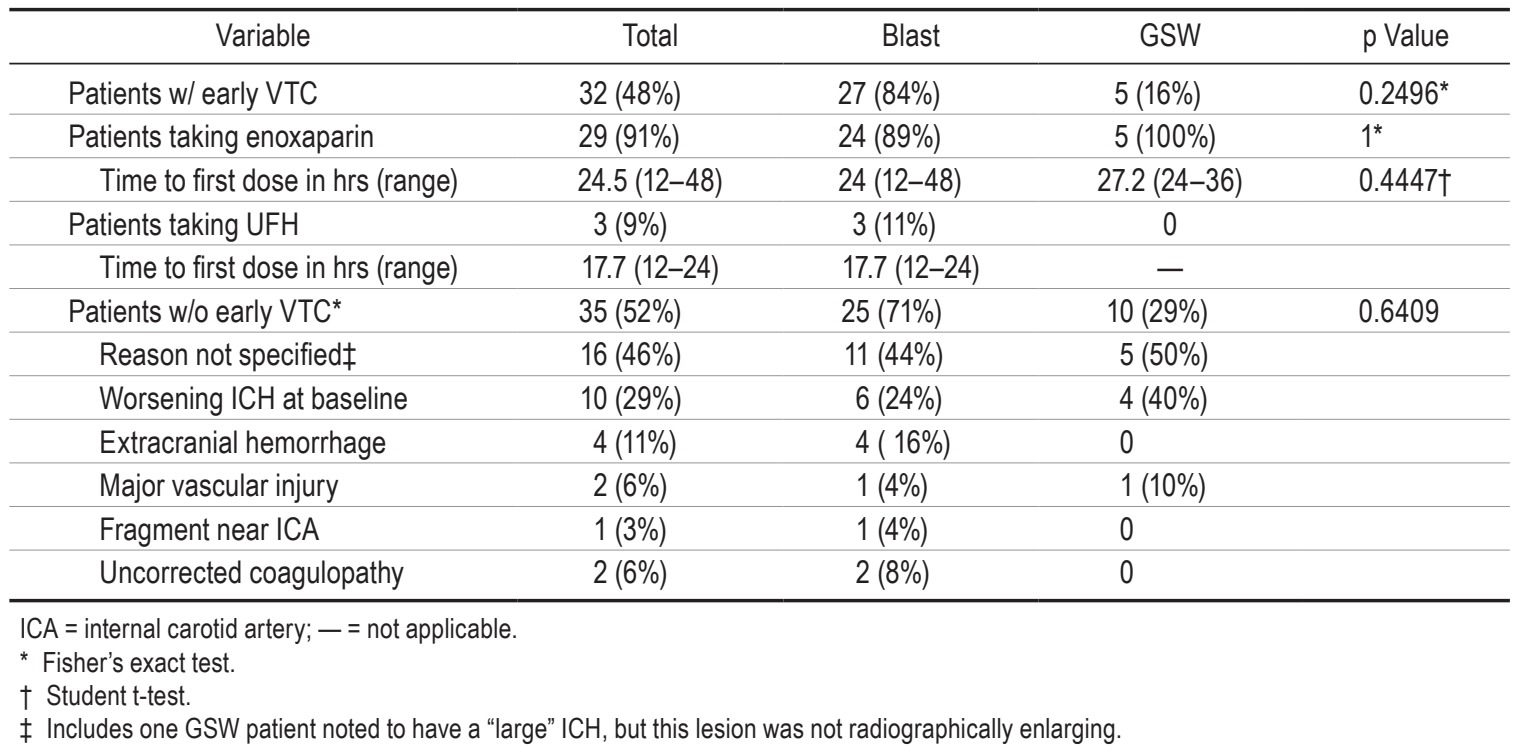

ses of overall and incident ICH progression rates, rate of DVT or PE, 30-day mortality, and nonelective reoperation were conducted for the blast subgroup in the same manner described above.

Supplemental Table 3 summarizes this outcome information for blast-injured patients who received early VTC compared with those who did not, which was similar to that for the overall cohort: incident-worsened ICH RR 0.93 (95\% CI 0.27-3.20), overall worse ICH RR 0.66 (95\% CI 0.21-2.08), DVT or PE RR 0.62 (95\% CI 0.17-2.19), 30day mortality RR 1.00 (95\% CI 0.06-16), and nonelective reoperation RR 0.46 (95\% CI 0.04-5.11). As with the full cohort, these associations did not significantly change after accounting for aGCS score, ISS, and AIS score in the regression model.

\section{Discussion}

The purpose of this retrospective cohort study was to analyze the clinical course of active duty US military personnel who had sustained PBIs in combat and were subsequently treated by military neurosurgeons. The study population largely reflected the demographics of the US military, and the patterns of injury observed were similar to those noted in other recent studies of military TBI and PBI, in which blast has constituted the most prevalent mechanism of injury. ${ }^{1-4,7,16,17}$ The proportions of individuals who did and did not receive early VTC were evenly split, which reflects the current state of equipoise about the use of early prophylactic anticoagulation in brain-injured patients.

In this cohort, the administration of VTC within 12-48 hours of sustaining a PBI was not associated with worsening ICH or with a significant difference in the rate of DVT or PE. This intervention was also not associated with an increased rate of emergent reoperation or 30-day mortality.

Complications of hypercoagulability were significant morbidities in our population, with a combined rate of DVT and PE at $15 \%$. This incidence is higher than expect- ed given the results of a 2004 review of more than 450,000 trauma patients by Knudson et al., who found a rate of $0.36 \% .{ }^{11}$ The most significant of those hypercoagulability complications in our study were 2 cases of PE, which represent an incidence of 3\%. Again, this rate is high when considered in the context of that large review, in which the incidence of PE was $0.13 \%,{ }^{11}$ but the absolute number of incident PE in our study was low, limiting the interpretability of this figure. However, rates observed in our study are consistent with what has been previously noted in the PBI-in particular, the combat-related PBI-population. A 2009 study on the combat PBI population found the rate of PE to approach $7 \%,{ }^{3}$ and a study in 2012 found a $15 \%$ combined incidence of DVT and PE..$^{17}$

It is possible that combat-related PBI in active duty military personnel is fundamentally different from PBI in other, more heterogeneous populations and that, subsequently, the observed safety of early VTC in this cohort may reflect a pharmacologically facilitated return of dramatically increased coagulability to a more physiological level. One potential difference is the preponderance of blast as a mechanism of injury. Penetrating brain injury caused by GSW was associated with a worse AIS score, aGCS score, and possibly ISS than PBI caused by blast. These differences may be attributable to the greater energy transferred to brain parenchyma and vascular structures by high velocity (that is, high kinetic energy) bullets than by lower velocity (that is, lower kinetic energy) blast debris.

While there were statistically nonsignificant differences in the ISS, AIS score, and aGCS score between the patients who did and did not receive early VTC, the addition of mechanism of injury as a covariate in the regression models did not appreciably change the relative risks of ICH worsening, nonelective reoperation, or mortality. Moreover, post hoc analyses limited only to the blast-injured personnel revealed estimates of the relative risks for the primary outcomes of interest similar those obtained in analyses of the GSW- and blast-injured patients together. 
TABLE 4. Incidence of outcomes related to early VTC in PBI

\begin{tabular}{|c|c|c|c|c|c|c|c|c|c|}
\hline \multirow[b]{2}{*}{ Variable } & \multirow[b]{2}{*}{ Total No. (\%) } & \multirow{2}{*}{$\begin{array}{c}\text { No Early VTC } \\
\text { No. }(\%)\end{array}$} & \multirow{2}{*}{$\begin{array}{c}\text { Early VTC } \\
\text { No. }(\%)\end{array}$} & \multicolumn{3}{|c|}{ Unadjusted RR } & \multicolumn{3}{|c|}{ Adjusted* RR } \\
\hline & & & & RR & $(95 \% \mathrm{Cl})$ & p Value & RR & $(95 \% \mathrm{Cl})$ & $\mathrm{p}$ Value \\
\hline Incident-worsened $\mathrm{ICH} \dagger$ & $11(16 \%)$ & $6(17 \%)$ & $5(16 \%)$ & 0.91 & $(0.28-2.99)$ & 0.8783 & 0.76 & $(0.21-2.79)$ & 0.6792 \\
\hline All worse $\mathrm{ICH} \dagger$ & $15(22 \%)$ & $10(29 \%)$ & $5(16 \%)$ & 0.55 & $(0.19-1.60)$ & 0.2705 & 0.56 & $(0.17-1.85)$ & 0.3386 \\
\hline DVT or PE & $10(15 \%)$ & $6(17 \%)$ & $4(12 \%)$ & 0.73 & $(0.21-2.58)$ & 0.6246 & 0.88 & $(0.20-3.93)$ & 0.8699 \\
\hline 30-day mortality & $4(6 \%)$ & $3(9 \%)$ & $1(3 \%)$ & 0.36 & $(0.04-3.50)$ & 0.3822 & 1.50 & $(0.07-34.0)$ & 0.7983 \\
\hline Non-elective reoperation $\ddagger$ & $4(6 \%)$ & $3(9 \%)$ & $1(3 \%)$ & 0.36 & $(0.04-3.50)$ & 0.3822 & 0.72 & $(0.06-8.71)$ & 0.7977 \\
\hline
\end{tabular}

* Adjusting for mechanism of injury, aGCS score, ISS, and AIS score, region 1. Referent group in each model was no early VTC.

$\dagger$ The distinction between incident and all worse ICH is the exclusion of 4 cases of incremental change of ICHs that enlarged prior to deciding to give or hold VTC.

$\ddagger$ If reoperation was listed as "NA" or "no" then it was counted as "no reoperation required."

Ten patients demonstrated enlarging ICHs in Afghanistan before the decision to administer or hold VTC was made, which in all 10 cases led the treating physician to hold VTC. Four of these 10 patients had incremental enlargement of the same lesions after evacuation. Given the retrospective nature of our study, it is difficult to assess whether these interval changes represent the true incidence of outcome (worsened ICHs) or are instead the expected evolution of a pathology that predated the exposure (giving or holding early VTC). To account for this, we sought to analyze all cases of ICH with what we believe is the logical equivalent of an intent to treat analysis (that is, accounting for all worse ICHs observed after evacuation as "incident") and in a "per protocol" fashion that only considers new cases of worsened ICH (new lesions or enlarging lesions that were stable at the time of exposure) as "incident."

Interpreting the rate of worsened ICHs in the intent to treat fashion increases the incidence in the "no early VTC" group by 4 cases, while the incidence in the early VTC group remains unchanged. This may mask a potential danger of early VTC, which is a potential source of Type II error. Thus, interpreting the rate of worsening ICH in a per protocol fashion is more likely to reveal a potential danger of early VTC administration and, in our opinion, is the most conservative assessment of this intervention's safety. In either case, there was no increased risk of worsening ICH in the early VTC group, which is consistent with our conclusion that early VTC was safe in this cohort.

In this study we cannot definitively assess the efficacy of early VTC for the prevention of DVT or PE. There were more hypercoagulable events (DVT and PE together) in the group without early VTC, and PEs occurred only in patients who did not receive early VTC. However, these observed differences did not rise to statistical significance as in all cases the 95\% CI included 1. The observational design of our study precluded a priori identification of all relevant potential confounders; therefore, the observed effects of early VTC administration could be attributable to the influence of 1 or more variables not included in these analyses.

The finding that the use of early VTC in patients with PBI was safe is consistent with the most recent systematic review of the literature examining this question in TBI patients in general, which is described in our introduction above..$^{15}$ The data regarding this intervention's role in preventing DVT and PE are also consistent with findings in that systematic review, which was unable to definitively show efficacy even after consideration of the full body of evidence currently in the literature..$^{15}$

One potential confounder is the influence of attending neurosurgeon preference and clinical judgment on the treatment decisions made. The evolution of combat neurotrauma care at KAF provided a natural experiment that, by chance, allocated patients approximately equally to 2 different treatment groups (early VTC and no early VTC) over the studied period. The preponderance of the term "not specified" as the listed contraindication for VTC administration is indicative of this surgeon-driven allocation of patients, and we noted strong patterns of individual surgeon preference. A post hoc analysis of demographic and injury characteristics according to the reason early VTC was withheld did not reveal any statistically significant associations that might indicate underlying confounding. Given these findings, we elected to not include attending neurosurgeon as a potential covariate because the very small number of neurosurgeons whose treatment decisions were analyzed in this study precludes adequate anonymization. However, unrecorded differences in the perioperative management of these patients according to treating neurosurgeon preferences may still be present, as is the case for any study that uses an observational design.

Factor VII and TXA exposure data were analyzed to assess for a potential confounding effect of these 2 procoagulant drugs, which were frequently used in the management of trauma patients at KAF. In this cohort there was no apparent association between these exposures and complications of bleeding or hypercoagulability.

Aside from the observational design, this study has other important limitations to note. The sample size, while comparable to those in many neurotrauma studies, is small. It is possible that some of the observed trends and differences in this study would reach thresholds of statistical significance with a more highly powered cohort. There is also considerable selection bias. The sample is exclusively male; comprises otherwise healthy, fit, active duty military personnel whose physiological reserve is probably greater than could be expected from age- and sexmatched controls from a nonmilitary population; and is certainly different from the US population at large. While this selection bias does limit the generalizability of our results, we believe that the data remain relevant given the 
fact that young males are disproportionately victims of trauma, including modes of injury that lead to PBI.

Our findings can only be definitively applied to the 67 patients in this cohort. With caution, it may be reasonable to generalize them to other military patients with PBI presenting in a clinically similar manner. Generalizing these findings to other PBI patients, such as those seen at civilian trauma centers, should be undertaken with extreme caution; examination of Tables 1 and 2 reveals that the study cohort is probably very different from what many readers may see in their civilian practices. However, given the dearth of alternative data, we believe that this study maintains some relevance to neurosurgeons managing PBI patients of any sort. At a minimum, the 5 specific features listed in Table 3-worsening ICH at baseline, ongoing clinically significant extracranial hemorrhage, injury to or an intracranial fragment that threatens to injure a major intracranial vessel, and coagulopathy not corrected by resuscitation-should probably be considered relative contraindications to early VTC after PBI. Moreover, since no reason was specified for withholding early VTC from $46 \%$ of the individuals in our cohort, it is imperative for neurosurgeons to carefully consider every patient on a case by case basis, especially when the listed relative contraindications are not present, until more stringent data exist to advise definitive practice guidelines.

To our knowledge this study is the only one to directly examine the use of early VTC in patients with PBIs. Thus, the data reflect the current state of equipoise regarding the safety and efficacy of early anticoagulation in neurotrauma patients. More importantly, these data indicate that early administration of VTC was safe in this cohort and is probably effective for preventing DVT and PE and should be considered as support for the establishment of well-designed, multicenter randomized controlled trials to definitively address this clinically important question.

\section{Conclusions}

Early administration of VTC was safe in this cohort of combat-related PBI patients with regard to progression of ICH. The data suggest that this intervention may have been effective in preventing DVT and PE, but not in a statistically significant way. More research is needed to clarify the safety and efficacy of this practice, particularly in other (that is, nonmilitary) patient populations.

\section{Acknowledgments}

We thank the 13 servicemen in our cohort who gave their lives and were beyond help when they presented to our care in Kandahar, the 4 who succumbed to their devastating brain injuries shortly after they were evacuated from Afghanistan, the many thousands of other servicemen and -women who have died in the service of the United States of America, and all of their families for their sacrifices. We also thank Steve Cobry, Min S. Park, Robert Rosenbaum, Dennis Rivet, and William Yarde, United States Navy neurosurgeons who served at Kandahar Airfield, Afghanistan, along with coauthors C.J.N., J.M.T., and R.S.B. during the violent era that includes our study period. Finally, we thank the other physicians, nurses, combat medics and corpsmen, technicians, and other medical professionals who cared for our patients on the battlefield, in field hospitals in Afghanistan, at Landstuhl Regional Medical
Center in Germany, at military hospitals in the United States, and aboard the aircraft transporting them in between.

\section{References}

1. Armonda RA, Bell RS, Vo AH, Ling G, DeGraba TJ, Crandall B, et al: Wartime traumatic cerebral vasospasm: recent review of combat casualties. Neurosurgery 59:1215-1225, 2006

2. Bell RS, Mossop CM, Dirks MS, Stephens FL, Mulligan L, Ecker R, et al: Early decompressive craniectomy for severe penetrating and closed head injury during wartime. Neurosurg Focus 28(5):E1, 2010

3. Bell RS, Vo AH, Neal CJ, Tigno J, Roberts R, Mossop C, et al: Military traumatic brain and spinal column injury: a 5 -year study of the impact blast and other military grade weaponry on the central nervous system. J Trauma 66 (4 Suppl):S104-S111, 2009

4. Bell RS, Vo AH, Roberts R, Wanebo J, Armonda RA: Wartime traumatic aneurysms: acute presentation, diagnosis, and multimodal treatment of 64 craniocervical arterial injuries. Neurosurgery 66:66-79, 2010

5. Bullock MR, Povlishock JT (eds): Guidelines for the management of severe traumatic brain injury, ed 3. J Neurotrauma 24 (Suppl 1):S1-S106, 2007

6. Denson K, Morgan D, Cunningham R, Nigliazzo A, Brackett $\mathrm{D}$, Lane M, et al: Incidence of venous thromboembolism in patients with traumatic brain injury. Am J Surg 193:380384, 2007

7. Ecker RD, Mulligan LP, Dirks M, Bell RS, Severson MA, Howard RS, et al: Outcomes of 33 patients from the wars in Iraq and Afghanistan undergoing bilateral or bicompartmental craniectomy. J Neurosurg 115:124-129, 2011

8. Ekeh AP, Dominguez KM, Markert RJ, McCarthy MC: Incidence and risk factors for deep venous thrombosis after moderate and severe brain injury. J Trauma 68:912-915, 2010

9. Farooqui A, Hiser B, Barnes SL, Litofsky NS: Safety and efficacy of early thromboembolism chemoprophylaxis after intracranial hemorrhage from traumatic brain injury. J Neurosurg 119:1576-1582, 2013

10. Ingalls N, Zonies D, Bailey JA, Martin KD, Iddins BO, Carlton PK, et al: A review of the first 10 years of critical care aeromedical transport during Operation Iraqi Freedom and Operation Enduring Freedom: the importance of evacuation timing. JAMA Surg 149:807-813, 2014

11. Knudson MM, Ikossi DG, Khaw L, Morabito D, Speetzen LS: Thromboembolism after trauma: an analysis of 1602 episodes from the American College of Surgeons National Trauma Data Bank. Ann Surg 240:490-498, 2004

12. Phelan HA, Wolf SE, Norwood SH, Aldy K, Brakenridge $\mathrm{SC}$, Eastman AL, et al: A randomized, double-blinded, placebo-controlled pilot trial of anticoagulation in low-risk traumatic brain injury: The Delayed Versus Early Enoxaparin Prophylaxis I (DEEP I) study. J Trauma Acute Care Surg 73:1434-1441, 2012

13. Reiff DA, Haricharan RN, Bullington NM, Griffin RL, McGwin G Jr, Rue LW III: Traumatic brain injury is associated with the development of deep vein thrombosis independent of pharmacological prophylaxis. J Trauma 66:1436-1440, 2009

14. Scudday T, Brasel K, Webb T, Codner P, Somberg L, Weigelt J, et al: Safety and efficacy of prophylactic anticoagulation in patients with traumatic brain injury. J Am Coll Surg 213:148-154, 2011

15. Shen X, Dutcher SK, Palmer J, Liu X, Kiptanui Z, Khokhar $\mathrm{B}$, et al: A systematic review of the benefits and risks of anticoagulation following traumatic brain injury. J Head Trauma Rehabil 30:E29-E37, 2015

16. Stephens FL, Mossop CM, Bell RS, Tigno T Jr, Rosner MK, Kumar A, et al: Cranioplasty complications follow- 
ing wartime decompressive craniectomy. Neurosurg Focus 28(5): E3, 2010

17. Weisbrod AB, Rodriguez C, Bell R, Neal C, Armonda R, Dorlac W, et al: Long-term outcomes of combat casualties sustaining penetrating traumatic brain injury. J Trauma Acute Care Surg 73:1525-1530, 2012

\section{Disclosures}

The authors report no conflict of interest concerning the materials or methods used in this study or the findings specified in this paper.

\section{Author Contributions}

Conception and design: Bell, Meyer. Acquisition of data: Meyer, Larkin. Analysis and interpretation of data: Bell, Meyer, Larkin, Szuflita. Drafting the article: Meyer, Larkin, Szuflita. Criti- cally revising the article: Bell, Neal, Tomlin, Armonda, Bailey. Reviewed submitted version of manuscript: all authors. Approved the final version of the manuscript on behalf of all authors: Bell. Statistical analysis: Szuflita. Administrative/technical/material support: Meyer. Study supervision: Bell.

\section{Supplemental Information}

Online-Only Content

Supplemental material is available with the online version of the article.

Supplemental Tables. http://thejns.org/doi/suppl/10.3171/ 2016.4.JNS16101.

\section{Correspondence}

Randy S. Bell, Division of Neurosurgery, Department of Surgery, Uniformed Services University of the Health Sciences and Walter Reed National Military Medical Center, 8901 Wisconsin Ave., Bethesda, MD 20889. email: randy.s.bell.mil@mail.mil. 